\title{
THE FORMATION AND REACTIONS OF 9,10-PHENANTHRYNE AND RELATED ARYNES BY PYROLYTIC REACTIONS IN THE VAPOR PHASE
}

\author{
Hans-Fr. Grǘzmacher* and Udo Straetmans \\ Fakuthảt für Chemie, Universităt Bielefeld, Universitätsstrasse, D-4800 Bielefeld 1, Germany
}

(Rectioed in Germany 23 April 1979)

\begin{abstract}
Abdract-The formation of 9,10-phenanthryne (5), 4methyl-9,10-phenanthryne (7) and 9,10-benz(c)phenanthryne (9) by the thermal degradation of appropriate arenedicarboxylic acid anhydrides has been investigated by a combination of VLPP and mass spectrometry and by a co-pyrolysis of the anhydrides with benzene, hexadeuterobenzene and 1,3-butadiene. Compounds 5,7 and especially 9 are formed easily by VLPP at 700-900 from the anhydrides. The results indicate that 7 and 9 are less reactive by $\mathrm{H}$-addition to the aryne bond than 5 , benzyne or naphthalyne, respectively. The results of the co-pyrolysis experiments show that 5 and 9 react with benzene and 1,3-butadiene by $\mathrm{H}$-abstraction and addition reactions, similar to 1,2-benzyne and 2,3-naphthalyne. However, the reactivity of the aryne in the $\mathrm{H}$-abstraction reaction decreases and the selectivity for the addition reaction increases in the series 1,2-benzyne, 2,3-naphthalyne, 9,10-phenanthryne and 9,10-benz(c)phenanthryne.
\end{abstract}

The combination of very low pressure pyrolysis (VLPP) and mass spectrometry (MS) has been proved to be a very convenient method to study the formation of bisdehydroarenes (arynes) from certain disubstituted aromatic compounds. ${ }^{-6}$ By this technique ${ }^{36}$ the vapor of the substance to be pyrolysed is introduced into the ionisation chamber of a mass spectrometer through a quartz capillary, which is located within the ion source housing and which can be electrically heated to temperatures of $1000^{\circ}$. Thus the molecules of the precursor substance are pyrolysed at a pressure below $10^{-4}$ Torr just before entering the ion source and primary pyrolysis products can be detected after ionisation in the mass spectrometer. If the energy of the ionising electron beam is kept low enough, only the molecular ions of the precursor substance and of its pyrolysis products, respectively, are observed in the mass spectrum. Neglecting any differences in the ionisation cross sections, the intensity ratios of the signals in the mass spectrum roughly indicate the relative yields of the pyrolysis products. Furthermore observing the reduction in the intensity of the molecular ions of the precursor by switching on the pyrolysis reactor to the appropriate temperature, gives information about the sensitivity of the substance towards VLPP. This intensity reduction, expressed as percentage of the ion intensity without pyrolysis, will be quoted as pyrolysis yield.

During our investigation of several benzynes, ${ }^{2}$ naphthalynes ${ }^{3}$ and phenanthrynes ${ }^{4}$ by VLPP-MS it was observed, that 9-mono- and 9,10-disubstituted phenanthrene derivatives are much more easily pyrolysed than the corresponding benzenes and naphthalene derivatives and form 9,10-phenanthryne in high yields. In this paper some further experiments on the formation of substituted 9,10-phenanthrynes by VLPP and their reaction with benzene and 1,3-butadiene are reported.

VLPP-MS-experiments. Figs. 1-3 show the mass spectra of the pyrolysis products obtained by VLPP-MS of phenanthrene-9,10-dicarboxylic acid anhydride (1), 4 methyl-phenanthrene-9,10-dicarboxylic anhydride (2) and benz(c)phenanthrene-9,10-dicarboxylic anhydride (3), respectively.

The anhydrides of aromatic ortho-dicarboxylic acids

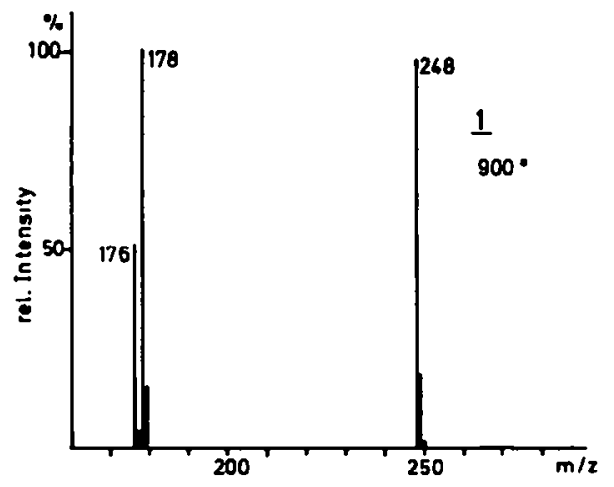

Fig. 1.

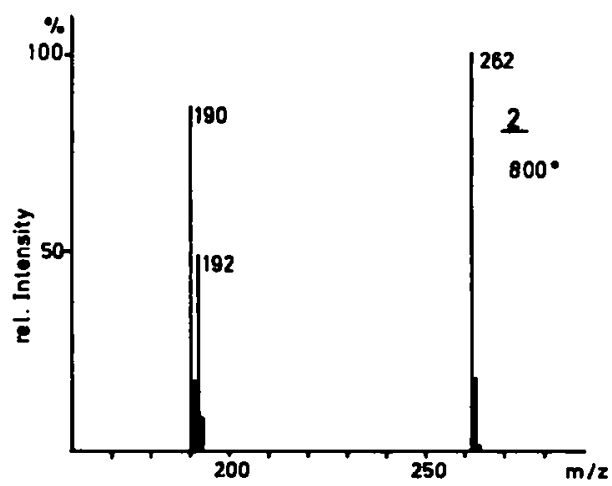

Fig. 2.

have been shown to be excellent precursors for the formation of the corresponding arynes on pyrolysis ${ }^{3}$ and the thermal degradation of 1 has been investigated by the VLPP-MS-methods before. ${ }^{4}$ The present results (Fig. 1) are in very good agreement with these earlier observations. The pyrolysis yield of 1 at $8000^{\circ}$ under the experimental conditions used, is about $60 \%$. Besides the peak at $\mathrm{m} / \mathrm{z} 248$, due to molecules of 1 surviving the VLPP, only two pyrolysis products of molecular weights ( = MW) 178 and 176, respectively, are indicated by large 


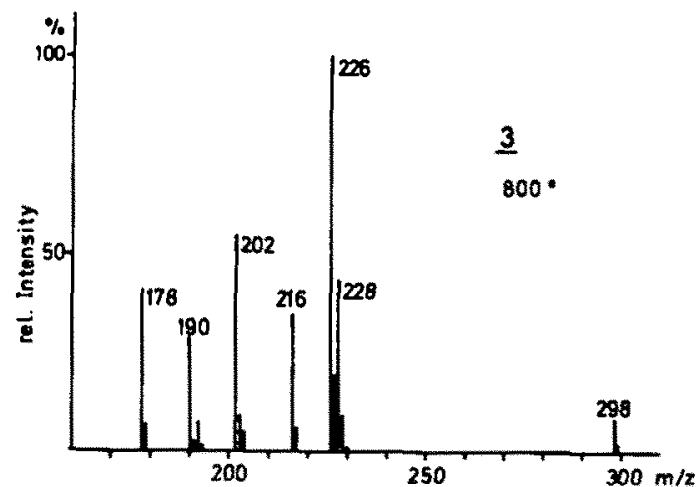

Fig. 3.

peaks in the spectrum. According to the MW's these products are phenanthrene (4) and 9,10-phenanthryne (5). These assignments are corroborated by an observation of the decay time of the signals of the products in the mass spectrum after switching off the beating of the pyrolysis reactor and by measurements of the ionisation energies (I(M)).

During the VLPP-experiment there is a steady state concentration of unstable reactive pyrolysis products and stable unreactive ones in the gas phase of the ionisation chamber. If the pyrolysis is stopped by switching of the electrical heating of the reactor, the rate of decay of the signals of stable unreactive pyrolysis products is determined by the rate of diffusion out of the ionisation chamber and ion source housing of the mass spectrometer. Typical decay times $\tau_{1 / 2}$ for the signals of unreactive products to drop to $50 \%$ of their original values are $15-45 \mathrm{~s}$. However, the decay times $\tau_{1 / 2}$ for unstable reactive pyrolysis products are much shorter being about $1-2 s$ because these species disappear mainly by fast chemical reactions in the gasphase and at the walls of the ion source.

In the VLPP of $1, \tau_{1 / 2} \approx 2 \mathrm{~s}$ and $r_{1 / 2}>20 \mathrm{~s}$ are observed for the products $\mathrm{MW} 176$ and 178 , respectively, as expected for the unstable compound 5 and the stable hydrocarbon 4. The ionisation energies of these products, determined by the RPD-method, ${ }^{6}$ are $\mathrm{I}(176)=$ $8.0 \pm 0.1 \mathrm{eV}$ and $I(178)=8.0 \pm 0.1 \mathrm{eV}$, in good agreement with the values reported in the literature for $5^{4}$ and $4^{7}$ Therefore it can be concluded, that 1 looses $\mathrm{CO}_{2}$ and $\mathrm{CO}$ in a clean reaction during VLPP to give 5 as the primary pyrolysis product, which picks up two hydrogens $f$ during its flight from the pyrolysis zone to the ionisation chamber and forms as a secondary pyrolysis product (Scheme 1).

The VLPP of 2 (Fig. 2) at temperatures of $700-800^{\circ}$ gives apparently only two products of $\mathrm{MW} 190$ and $\mathrm{MW}$ 192 the pyrolysis yield being again $\mathrm{ca} .60 \%$. The rather long decay time $\tau_{1 / 2}>20 \mathrm{~s}$ and the $\mathrm{I}(192)$ of $7.8 \pm 0.1 \mathrm{eV}^{7}$ identifies the product $\mathrm{MW} 192$ as 4-methylphenanthrene (0). Correspondingly one will expect the product MW 190 to be 4 -methyl-9,10-phenanthryne (7); however, 4,5-

TPart of 1 is more or less completely destroyed by VLPP at $800-1000^{\circ}$, as is indicated by coating of the quartz capillary of the pyrolysis reactor with carbonous material after the experiments. Obviously hydrogen is set free by this complete degradation and the hydrogenation of arynes to the corresponding arene has been observed in all our experiments. $2-4$

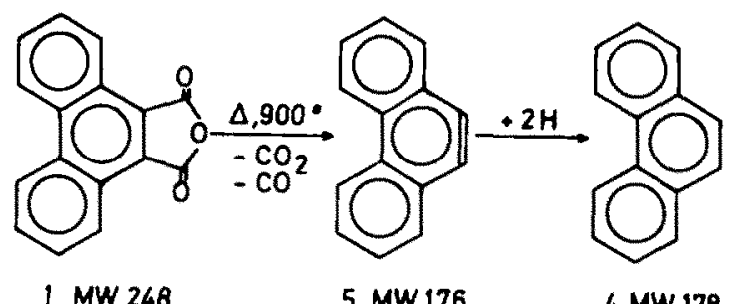

Scheme 1.

methylenphenanthrene(8), formed by loss of $\mathrm{H}_{2}$ and ring closure from 4methyl-phenanthrene, is also a likely candidate. Between both possibilities can be differentiated by observing the decay time of the signal at $\mathrm{m} / \mathrm{e}$ 190, because 8 is expected to be stable under the experimental conditions, whereas 7 is a reactive primary pyrolysis product. The observed value, $\tau_{1 / 2} \approx 12 \mathrm{~s}$, is typical for an aryne, and as long as the pyrolysis temperature is kept below $800^{\circ}$, the signal at $\mathrm{m} / \mathrm{z} 190$ disappears completely in a few seconds after switching of the pyrolysis. Thus by far most of the signal at $\mathrm{m} / \mathrm{z} 190$ observed at a pyrolysis temperature of $800^{\circ}$ is due to 7 . This is of interest because contrary to the intensity ratio of $5 / 4$ (Fig. 1), the intensity of 7 exceeds that of the corresponding arene 6 . Although the intensities in the mass spectra obtained by VLPP-MS vary somewhat even under apparently constant experimental conditions, this different behaviour between 5 and its $4 \mathrm{Me}$ derivative 7 is always observed. Obviously hydrogenation of 7 is not as easy as that of the parent aryne 5 . The ionisation energy $7.7 \pm 0.1 \mathrm{eV}$ of 7 is only slightly below that of 6 , as it was the case with the couple 5/4.

If the VLPP of 2 is performed at temperatures above ca. $800^{\circ}$, the signals of two additional pyrolysis products appear at $\mathrm{m} / \mathrm{z} 176$ and $\mathrm{m} / \mathrm{z} 178$. At the same time the intensity of the signal at $\mathrm{m} / \mathrm{z} 190$ rises, but now only a part of this signal disappears with a short decay time after switching of the pyrolysis reactor, while the remaining part of the signal decays rather slowly. This indicates the formation of a stable species MW 190 , which is presumably $\mathbf{8}$, in addition to 7 . The product $\mathbf{M W}$ 178 is 4 as shown by its $\tau_{1 / 2}>20 \mathrm{~s}$ and its ionisation energy. The product $\mathrm{MW} 176$ is an unstable reactive species which has the molecular weight of a phenanthryne and probably corresponds to 4,5-phenanthryne.' It has been demonstrated by VLPP-experiments with 4 methyl-phenanthrene 6 , that these three additional products arise from pyrolytic reactions of this compound at high temperatures. ${ }^{1}$ The pyrolytic reactions of 2 in VLPP are shown in Scheme 2.

The anhydride 3 is much more sensitive to pyrolysis than 1 and 2. The mass spectrum obtained during VLPP (Fig. 3) shows only a rather small peak of surviving molecules of 3 with MW 298; the pyrolysis yield being $>90 \%$. Products of MW's 228, 226, 216, 202, 190 and 178 are observed. As is shown by a typical short decay time $\tau_{1 / 2}$, only the compound MW 226 is a short lived primary pyrolysis product, corresponding to 9,10 benz(c)phenanthryne( 9 ). This is corroborated by the ionisation energy of $7.6 \pm 0.1 \mathrm{eV}$, which is close to the ionisation energy $7.76 \mathrm{eV}^{7}$ of benz(c)phenanthrene (10) and the addition of $\mathrm{H}_{2}$ to 9 to form 10, MW 228. As it was observed for 7 , the intensity of the signal of 9 at 226 always exceeds that of the parent hydrocarbon 10 at 228 (Fis. 3), indicating again a reduced reactivity of the aryne 
<smiles>Cc1cccc2c3c(c4ccccc4c12)C(=O)OC3=O</smiles>

2 MW262<smiles>Cc1cccc2ccc3ccccc3c12</smiles>

7 MW 190

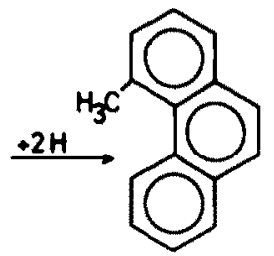

6 MW 192

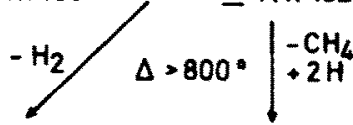

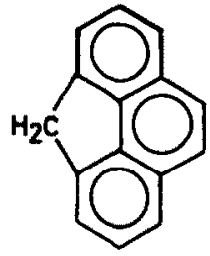

B $M W 190$

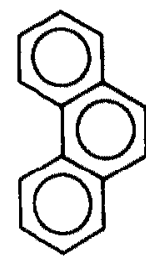

$4 M W 178$

Scheme 2

toward hydrogen addition. The structures of the other (stable) pyrolysis products have not been determined, so the structures shown in Scheme 3 are tentatively given.

Reactions with benzene and 1,3-butadiene. To get additional information about the reactivity of the arynes $\mathbf{s}$ and 9 the anhydrides 1 and 3 have been pyrolyzed in a stream of benzene, hexadeuterobenzene or 1,3butadiene, respectively, at temperatures of $c a .700^{\circ}$ and a total pressure of 10-20 Torr in a quartz tube. Under these conditions the pyrolysis of benzene and 1,3butadiene, respectively, as pure compounds, give only small amounts of polymeric material. About $50 \%$ of the materials pyrolysed in the co-pyrolysis experiments were recovered as high boiling destillates, which were analysed by gas chromatography and a GC/MS-combination. The results are shown in Tables 1 and 2 .

The co-pyrolysis of 1 and benzene results in the formation of biphenyl (11), phenanthrene (4) and triphenylene (12) as the main products besides some minor com- ponents. These include fluorene and 9-phenylphenanthrene, as shown by comparison of their GC retention times and mass spectra. The pyrolysis of 1 in the presence of $\mathrm{C}_{6} \mathrm{D}_{6}$ gives a similar mixture of the same compounds, which contained deuterium to various degrees as indicated in Table 1.

Similarly the co-pyrolysis of anhydride 3 and benzene gives 11, benz(c)phenanthrene (10) and benz(g)chrysene 13 the two last compounds being the main products. Compound 11 is only a minor component of this reaction mixture, and besides 11 there are quite a number of compounds formed in similar amounts which, however, could not be identified by GC/MS-analysis. The deuterium incorporation into the products by co-pyrolysis of 3 with $C_{6} D_{5}$ is shown also in Table 1.

If anhydrides 1 or 3, respectively, are pyrolysed in a stream of 1,3-butadiene, the reaction products shown in Table 2 have been observed. Compound 1 forms 4 , 9,10-dihydrophenanthrene (14) and 12 as the main

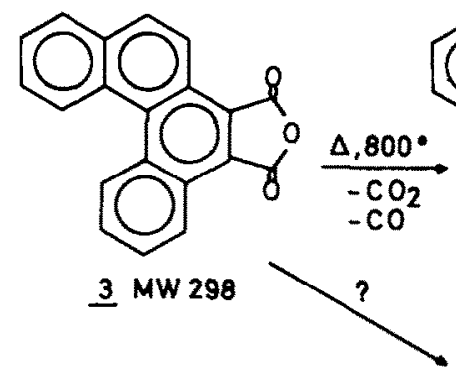

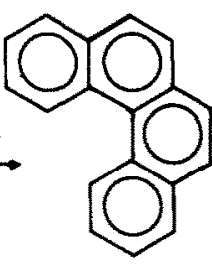

9. MW 226
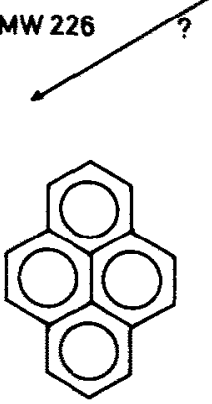

MW 202

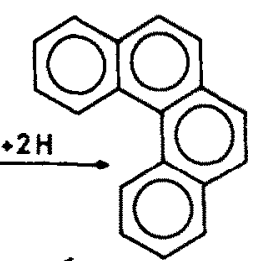

10 MW 228

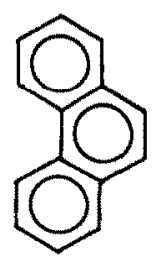

MW 178

Scheme 3. 
Table 1. Prochucts from pyrolysis of phenanthrenc-9,10-dicarboxylic anhydride (1) and benz(c)phenanthrene-9,10. dicarboxylic anhydride (3) in $\mathrm{C}_{6} \mathrm{H}_{6}$ and $\mathrm{C}_{6} \mathrm{D}_{6}$

\begin{tabular}{|c|c|c|c|}
\hline onbyatride & product & $\begin{array}{l}\text { rel.con- } \\
\text { centration al }\end{array}$ & deuterium content \\
\hline \multirow[t]{5}{*}{1} & biphenyl 11 & 1,0 & $>978 a_{10}$ \\
\hline & phenanthrene $\leq$ & 2,0 & $300 d_{2}, 400 d_{1}, 300 d_{0}$ \\
\hline & triphenylen 12 & 1,5 & $900 d_{4}, 100 d_{0}$ \\
\hline & $\begin{array}{l}\text { 9phenyl- } \\
\text { phenenthrene }\end{array}$ & $<0,2$ & $>978 d_{6}$ \\
\hline & fluorene & $<0,2$ & $258 d_{2}, 258 d_{1}, 500 d_{0}$ \\
\hline \multirow[t]{4}{*}{$\underline{\mathbf{3}}$} & bipheny 1 11 & 1.0 & $>978 d_{10}$ \\
\hline & $\begin{array}{l}\text { beme (c) phea- } \\
\text { anthrese } 10\end{array}$ & 7,0 & $282 d_{2}, 39 a_{1}, 328 d_{0}$ \\
\hline & bess (g) chrysene 13 & 10,0 & $\sim 958 d_{4}$ \\
\hline & phenanthrene 4 & 3,0 & - \\
\hline
\end{tabular}

a) estimated from peak heights in the gas chromatogram

Table 2. Products from pyrolysis of phenanthrene-9,10-dicarboxylic anbydride (1) and benz(c)phenanthrene-9,10dicarboxylic anhydride (3) in 1,3-butadiene

\begin{tabular}{|c|c|c|}
\hline ampatrida & product & rel.conoentration a) \\
\hline \multirow[t]{3}{*}{1} & phenanthrene 1 & 1,0 \\
\hline & $\begin{array}{l}\text { 9,10-dimytro- } \\
\text { phenentheen } 14\end{array}$ & 2,3 \\
\hline & triphenylene 12 & 3,5 \\
\hline \multirow[t]{2}{*}{$\underline{\mathbf{3}}$} & bere (c) phenentheeno 10 & 1,0 \\
\hline & bens (g) chrystan 13 & 10,0 \\
\hline
\end{tabular}

a) estinuted from pauk holghts in the gas chronatogran

products (ca 70\%) and only a few minor byproducts, which have not been identified. In the case of 3 about $80 \%$ of the mixture of volatile reaction products consists of 10 and 13. The rest of the mixture contains a number of compounds in low concentrations which have not been investignted.

\section{Dactusion}

Whereas disubstituted derivatives of benzene, naphthalene and phenanthrene form the corresponding bisdehydroarenes on pyrolysis at temperatures between 800 and $1000^{\circ}$ by loss of both substituents, ${ }^{24}$ there is a distinct difference in the pyrolytic fragmentations of monosubstituted benzenes and naphthalenes on one side and 9-substituted phenanthrenes on the other side. The only pyrolysis products observed in VLPP-MS experiments of chloro-, bromo- and iodo-benzenes and naphthalenes are the corresponding arenes, which are formed by loss of the halogenosubstituents and addition of a H-atom to the reactive phenyl or naphthyl radicals. ${ }^{34}$ In contrast to this 9-halogeno- and 9-nitrophenanthrene yield the aryne 5 as a primary pyrolysis product quite casily," comparable to the pyrolytic fragmentation of disubstituted derivatives. Thus it appears that $\mathbf{5}$ is for- med more easily and hence is probably more stable (less reactive) than 1,2-benzyne, 1,2-naphthalyne and 2,3naphthalyne.

Enhanced selectivity and correspondingly less reactivity has been observed for reaction of $\boldsymbol{S}$ in solution and has been explained by a shielding of the reactive "aryne" bond of $\mathbf{5}$ by $\mathrm{H}$-atoms at the peri-position." This effect, bowever, can not explain the greater ease of formation of $\mathbf{S}$ by pyrolytic fragmentations. Furthermore the high yield of an aryne as a primary product on pyrolysis of monosubstituted arenes appears to be an unique property of phenanthrenes, as no didehydropyrene is observed during the VLPP of 3 - and 4bromopyrene, the only product detected being pyrene.' Thus in spite of the possibility to form an aryne with an extended $\pi$-system and a chemical surrounding at the aryne bond similar to 5, the substituted pyrenes behave like substituted naphthalenes during pyrolysis.

Theoretical calculations of the geometry of 1,2-benzyne have shown, ${ }^{10}$ that in this molecule the carbon skeleton of the benzene nucleus is distorted to allow for a short bonding distance of the "aryne" bond between C-1 and C-2. Similar deviations from the geometry of the carbon skeleton of the parent arene are to be expected 
for the other arynes. Possibly the rearrangement of the carbon skeleton during the transformation of a phenanthrene derivative into 9,10-phenanthryne 5 is assisted by a "back strain" in the phenanthrene molecule due to a repulsion between the $\mathrm{H}$-atoms at position 4 and 5 , "which is relieved by shortening the $\mathrm{C}-9-\mathrm{C}-10$ bonding distance. This effect would explain the greater tendency of 9-substituted phenanthrenes to form 5 during pyrolysis, in contrast to naphthalene and pyrene derivatives, and may also be of some importance for the reduced reactivity of 5 , since an addition to the aryne bond will restore the strain in the resulting phenanthrene derivative.

If this explanation is correct, the 4substituted 9,10 phenanthrynes 7 and 9 should be formed even more easily and should be less reactive than 5 , because of the increased steric strain in 4-substituted phenanthrenes. The VLPP-experiments with the anhydrides 1,2 and 3 show, that the pyrolytic fragmentation of 2 proceeds at least as easily and that of $\mathbf{3}$ much more easily than that of 1, the arynes 5, 7 and 9, respectively, being the main primary pyrolysis product. As can be seen from the mass spectra of the products shown in Figs. 1-3, 7 and 9 show a remarkable small tendency to form the parent arene 6

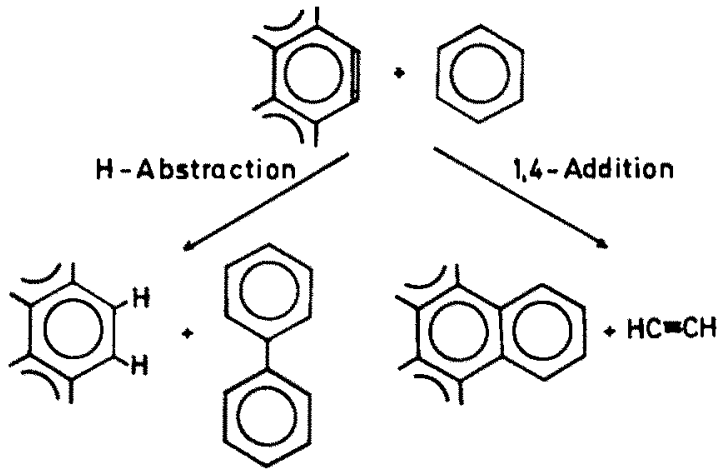

Scheme 4.

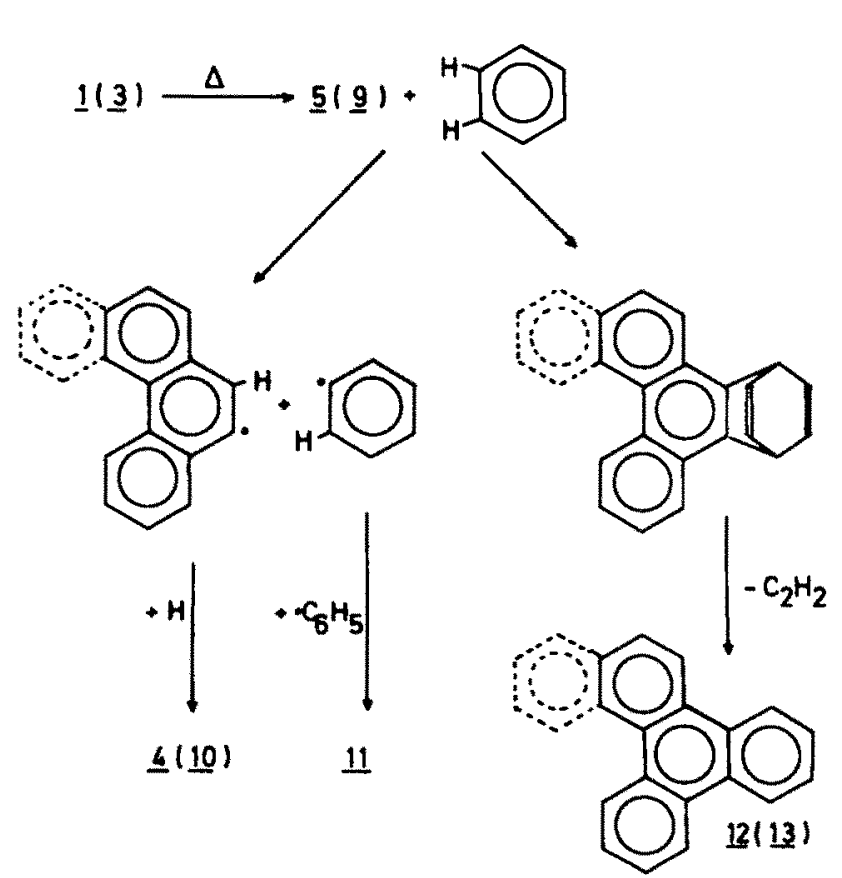

Scheme 5 . and 10 , respectively, by addition of $\mathrm{H}$-atoms to the aryne bond. Under comparable experimental conditions the ratio of the intensities of aryne and arene are 0.5 for $3 / 4$, 1.8 for $7 / 6$ and 2.2 for $9 / 10$, indicating a reduced reactivity of 7 and 9.

Additional informations about differences in the reactivity of 5 and 9 are obtained by the pyrolysis of 1 and 3 in the presence of benzene, hexadeuterobenzene and 1,3-butadiene, respectively. The reactions of arynes, generated by a pyrolytic fragmentation of the appropriate aromatic dicarboxylic acid anhydrides, with $\mathrm{C}_{6} \mathrm{H}_{6}$ and $\mathrm{C}_{6} \mathrm{D}_{6}$ have been studied by Fields and Meyerson. The two main reactions are a $\mathrm{H}$-abstraction from $\mathrm{C}_{6} \mathrm{H}_{6}$ by the aryne, which gives biphenyl 11 and the corresponding arene as the final products, and a 1,2- or more probably 1,4-addition of the aryne to $\mathrm{C}_{6} \mathrm{H}_{6}$, a benzannelated arene being the final product (Scheme 4).

The high boiling pyrolysate, obtained by a co-pyrolysis of phthalic anhydride and benzene, contained as a major portion a mixture of $\mathbf{1 1}$ and naphthalene in the relative concentrations of 1.00:0.18. ${ }^{3}$ In similar experiments with naphthalene-2,3-dicarboxylic anhydride the pyrolysate contained 11, naphthalene, anthracene and phenylnaphthalene in the relative concentrations $1.00: 0.44: 0.85: 0.68$. It is of interest to note, that relative to 11, which is the typical product of the $\mathrm{H}$-abstraction reaction, the amount of the addition product anthracene is considerably larger in the case of 2,3naphthalyne. Thus it appears that 2,3-naphthalyne reacts more selectively, favouring the addition reaction.

This effect is also observed in our experiments. The main products in the co-pyrolysis of 1 and benzene and hexadeuterobenzene, accounting for about $90 \%$ of the volatile high boiling products, are 11,4 and 12 in the relative concentrations $1.0: 2.0: 1.5$. The deuterium content of 11 was $>97 \% d_{10}$ in the experiment with $C_{6} D_{6}$. This clearly shows, that 11 is formed from benzene by the $\mathbf{H}$-abstraction reaction, as proposed by Fields and Meyerson' (Scheme 5). Since in the same experiment the deuterium content of 4 was $30 \% d_{2}, 40 \% d_{1}$ and $30 \% d_{0}$, 
the other primary product of the H-abstraction reaction, the 9-phenanthryl radical, either azain abstracts a $\mathrm{H}$ atom from a benzene molecule in the gas phase or picks up a H-atom from the walls of the reactor or from other sources. Triphenylene 12 is the expected product of the 1,4addition of 5 to benzene (Scheme 5) and the deuterium content of 12, formed by the reaction with $C_{6} D_{6}$. was $90 \% \mathrm{~d}_{\text {, }}$, indicating a rather specific reaction without much $H / D$ exchange.

The co-pyrolysis of 3 and benzene gives only two main products, 10 and 13 (Table 1), accounting for about $66 \%$ of the volatile products. The rest of the pyrolysate consists of a mixture of compounds, which have not been identified by the GC/MS-analysis, besides 4 and 11 . The relative concentrations of 11,10 and 13 are 1.0:7.0:10.0. The deuterium contents of these products, formed by reaction with $C_{6} D_{6}$, show the patterns expected by the reaction of Scheme 4. Again the deuterium content of 11 is $>97 \% \mathrm{~d}_{10}, 13$ contains about $95 \% \mathrm{~d}_{4}$, while 10 shows a distribution of $28 \% \mathrm{~d}_{2}, 39 \% \mathrm{~d}_{1}$ and $32 \%$ do.

The high yields of $11-d_{30}$ and $4 d_{4}$ or 13-d, respectively, in the reactions of 5 and 9 with $\mathrm{C}_{6} \mathrm{D}_{6}$ show, that the relative concentrations of these products can be used to characterise the selectivity of an aryne toward the H-abstraction and addition reaction with benzene. Including the results of Fields and Meyerson, which have been obtained under similar experimental conditions, the relative concentrations of 11 and the addition products are approximately $1: 0.2 ; 1: 0.9 ; 1: 1.5$ and $1: 10$ in the series 1,2-benzyne, 2,3-benzyne, 2,3-naphthyne, 9,10phenanthryne (5) and 9,10-benz(c)phenanthryne (9). Obviously the ability of the aryne to abstract a $\mathrm{H}$-atom from benzene decreases in this series, in agreement with the results of the VLPP-MS experiments, where a large signal for the aryne is observed for 7 and 9 (Figs. 2 and 3). Nevertheless the reactivity of all arynes investigated is large enough for 1,4addition reactions with benzene and other substrates. The selectivity of 9 in these reactions is also seen from the results of co-pyrolysis of 1 and 3, respectively, with 1,3-butadiene. The main products in the reaction of 5 , formed by fragmentation of 1, are 12,4 and the dihydro derivative 14 in the relative concentrations of about 1.0:0.3:0.7. 12 is formed by 1,4addition of 5 to 1,3-butadiene and dehydrogenation of the addition product (Scheme 6), while 4 is formed by H-abstraction reactions of 5 .

The pyrolysate obtained from the pyrolysis of 3 and 1,3-butadiene contained 70\% of 13, the product expected from 1,4-addition of to 1,4-butadiene and dehydrogenation, and only a small amount of 10 , the relative concentrations of 13 and 10 being 1.0:0.1. Thus the addition reaction of predominates over the $\mathrm{H}$-abstraction reaction again by a factor of 10 .

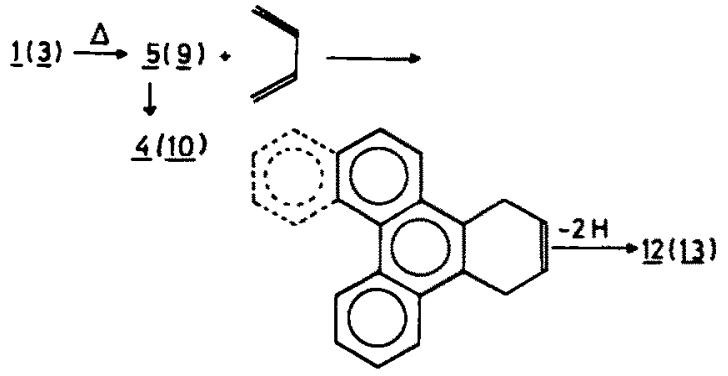

Scheme 6.
The observation, that certain arynes related to 9,10 phenanthryne are easily formed by pyrolytic reactions and possess a high reactivity and selectivity in addition reactions with arenes and unsaturated hydrocarbons, may be important with respect to the generation of polynuclear aromatic compounds during the combustion of hydrocarbons. If arenes containing only a few aromatic rings are formed by condensation of small hydrocarbon molecules and radicals ${ }^{5}$ during the combustion, the temperatures in these flames are sufficiently high for a formation of arynes by dehydrogenation processes or by elimination of small sidechains. By the high yield of addition products in the reaction of aryne 9 with benzene and butadiene one can expect similar addition reactions of these arynes with other aromatic compounds. This would be a very efficient mechanism for the formation of large polynuclear aromatic hydrocarbons from relatively small molecules by only a few reaction steps. It will be of interest to test this possibility by a further investigation of the formation and reactions of certain polynuclear arynes.

\section{Dxpingirara}

Compounds $1,{ }^{12} 2^{12}$ and $3^{13}$ have been synthesised according to the lit. The apparatus used for VLPP and its combination with a mass spectrometer have been described before." The quartz capillary of the reactor was activated by treatment with $\mathrm{CrO}_{3} / \mathrm{conc}$. $\mathrm{H}_{2} \mathrm{SO}_{4}$, thoroughly washing with $\mathrm{H}_{2} \mathrm{O}$ and heating to $900^{\circ}$ in vacuo. The pyrolysis of anhydrides 1-3 were performed at a pressure $<10^{-4}$ Torr and temps between $500^{\circ}$ and $900^{\circ}$ (as indicated in Figs. 1-3). The contact times of the molecules in the reactor are estimated to be $10^{-3}-10^{-2} \mathrm{~s}$. The mass spectra of the pyrolysis products were obtained at an (nominal) electron energy of $10 \mathrm{eV}$.

The ionisation energies have been determined by measuring the ionisation efficiency curves of the molecular ions of the products during a VLPP experiment with a Fox-ion source (Varian MAT, Bremen) by the retarding potential difference method using naphthalene $(\mathrm{I}(\mathrm{M})=8.26 \mathrm{eV})$ or phenanthrene $\left(I(M)=8.03 \mathrm{eV}^{7}\right)$ as an internal standard for the calibration of the electron energy.

The preparative co-pyrolysis of the anhydrides 1-3 with benzene, hexadeuterobenzene or 1,3-butadiene were performed in a quartz tube of $1 \mathrm{~cm}$ i.d., which was electrically heated at a length of $10 \mathrm{~cm}$ to $800^{\circ} .1 \mathrm{~g}$ of the compound to be pyrolysed was slowly evaporated by heating into a stream of the reagent at a pressure of 10-20 Torr just before the pyrolysis zone of the reactor, and the products were collected in a trap cooled by liquid $\mathrm{N}_{2}$. The condensed material was dissolved in benzene and treated with NaOHaq to extract unreacted anhydrides. The soln was concentrated in vacuo and the residue analysed by gas chromatography (Fa. Carto Erba, 2101 column $3 \%$ ov 17, 2m) or a GC/MSinstrument (Fa. Varian MAT III). The deuterium content of the products was calculated from the mass spectra obtained by the GC/MS-analysis.

The amount of the pyrolysate obtained varied in different runs. Typically about $50 \%$ (by weight) of the material evaporated in the pyrolysis was condensed in the trap, and about $50 \%$ of the condensate consisted of unreacted anhydrides.

Acknowledgement-We thank the Fonds der Chemischen Industrie for financial support of this work.

\section{DPidaces}

'U. Straetmans, Dissertation Universität Hamburg (1976).

${ }^{2} \mathrm{H}$. F. Grotzmacher and J. Lohmann, Liebigs Ann. 705, 81 (1967).

${ }^{30} \mathrm{H}$. F. Grützmacher and J. Lohmann, Ibid. 733, 88 (1970); 'H. F. Gritzmacher and W.-R. Lehmann, lbid. 2023 (1975).

4H. F. Grutzmacher and J. Lohmann, lbid 726, 47 (1969). 
${ }^{3}$ E. K. Fields and S. Meyerson, Ado. Phys. Ong. Chem. 6, 1 (1968).

'H. J. Schiff and G. G. Cloutier, J. Chim. Phys 31, 793 (1959).

'H. M. Rosenstock, K. Draxl, B. W. Steiner and J. T. Herron, J. Phys. Chem. Ref. Data 6. (19m) Suppl. No. 1.

Th. Kauffmann, H. Fischer and R. Nïmberg, Tetrahedron Letters 2911 (1967).
'U. Straetmans, Diplomarbeit, Universităt Hamburg (1973),

10J. O. Noell and M. D. Newton, J. Am. Chem. Soc. 101, S1 (1979).

"C. A. Coulson, Tetrahedron 19, 525 (1963).

${ }^{12}$ A. Jeanes and R. Adams. J. Am. Chem. Soc. 99,2608 (1937).

13. Samuszkovicz and E. J. Modest, Ibid. 2,566 (1950). 